\title{
Comparison of the Efficacy of Two Different Modified Release Methylphenidate Preparations for Children and Adolescents with Attention-Deficit/Hyperactivity Disorder in a Natural Setting: Comparison of the Efficacy of Medikinet ${ }^{\circledR}$ Retard and Concerta ${ }^{\circledR}$ - a Randomized, Controlled, Double-Blind Multicenter Clinical Crossover Trial
}

\author{
Manfred Döpfner, Ph.D., Claudia Ose, ${ }^{2}$ Roland Fischer, Ph.D., ${ }^{3}$ \\ Richard Ammer, M.D., Ph.D., ${ }^{3}$ and André Scherag, Ph.D. ${ }^{2}$
}

\begin{abstract}
Objective: The comparison of the efficacy of Medikinet ${ }^{\circledR}$ retard and Concerta ${ }^{\circledR}$ trial was a multisite, randomized, doubleblind, crossover trial that aimed at comparing the effects of two different modified release methylphenidate preparations (Medikinet retard: 50\% immediate release (IR); Concerta: 22\% IR) in a natural setting across the day in 113 randomized children and adolescents with attention-deficit/hyperactivity disorder (age range 6-16 years). The duration of the study per patient was 3 weeks.

Methods: The primary outcome variable was the German version of the "Swanson, Kotkin, Agler, M-Flynn, and Pelham scale" in the first 3 hours of school as assessed by teachers.

Results: Medikinet retard with a higher IR component than Concerta (and an equivalent daily dose) was superior to Concerta ( $p=0.0009)$, and Medikinet retard with similar IR components in the morning as Concerta (but a lower daily dose) was noninferior to Concerta with regard to the primary outcome. Further, exploratory analyses on teacher and parent ratings on attention-deficit/hyperactivity disorder and on externalizing symptoms during the day revealed no evidence for the superiority of Concerta over Medikinet retard in an equivalent daily dosage throughout the day.

Conclusion: Children and adolescents may be treated with a lower daily dose of Medikinet retard (which has a similar IR component as Concerta) without resulting in a clinically relevant worse effect during school time.
\end{abstract}

\section{Introduction}

A TTENTION-DEFICIT/HYPERACTIVITY DISORDER (ADHD) IS a clinically and etiologically heterogeneous disorder that is characterized by the core symptoms of a reduced concentration span, general motor restlessness, and lack of control of impulses. ADHD has a high prevalence rate worldwide (Faraone et al. 2003), and similar rates have been found in German representative surveys (Döpfner et al. 2008a). Follow-up studies showed that ADHD is associated with various long-term risks (Barkley 1998). It has been demonstrated that a carefully monitored and titrated stimulant therapy reduces not only the core symptoms but also the associated behavioral problems while increasing psychosocial functioning (Greenhill et al. 2001). Methylphenidate (MPH) is the most fre- quently used and best studied substance, and the robust effects of immediate release (IR) preparations and modified release preparations (MR) of MPH have been documented in meta-analyses (Banaschewski et al. 2006; Faraone et al. 2006; Faraone and Buitelaar 2010).

Medikinet $^{\circledR}$ retard and Concerta ${ }^{\circledR}$ are two MPH preparations with different release profiles and proved efficacy on ADHD symptoms as well as co-morbid conditions. Medikinet retard contains equal proportions of IR and slow release MPH. This oncedaily extended-release formulation had a duration of action of about 8 hours in an analog classroom setting, with efficacy corresponding to twice-daily administration of IR MPH (Döpfner et al. 2004). Efficacy of Medikinet retard has also been demonstrated in a natural setting (Döpfner et al. 2003) as well as in a long-term adult

\footnotetext{
${ }^{1}$ Department of Child and Adolescent Psychiatry and Psychotherapy, University of Cologne, Cologne, Germany.

${ }^{2}$ Institute for Medical Informatics, Biometry and Epidemiology, University of Duisburg-Essen, Essen, Germany.

${ }^{3}$ Medice, Iserlohn, Germany.

The study is funded by Medice Company.
} 
ADHD trial (Rösler et al. 2009). The tablet coating of Concerta dissolves within 1 to 2 hours and releases $22 \%$ of the total dose of $\mathrm{MPH}$. The remaining $78 \%$ of the dose is osmotically controlled (osmotic-release oral system [OROS] technology) and released over 10 hours through a lazer-drilled hole. The maximum effect occurs 6-8 hours after dosing. Evidence on the efficacy of Concerta was provided in an analog classroom setting (Sonuga-Barke et al. 2004; Swanson et al. 2004) and in natural settings (Wolraich et al. 2001; Stein et al. 2003; Wilens et al. 2006).

Although stimulants for the treatment of ADHD have been well studied, there have been few head-to-head comparisons of longacting drugs (Faraone and Buitelaar 2010). A few studies compared OROS MPH with other MPH preparations. Findling et al. (2008) compared the efficacy of MPH transdermal system with placebo and OROS MPH as reference therapy and found no evidence for differences between both active preparations on overall parent and teacher ratings. Due to the different pharmacokinetic profiles of the various MR MPH products, different time courses of action throughout the day are expected. The comparison of methylphenidates in the analog classroom setting (COMACS) study is the only study that has compared the pharmacodynamics of two different MR preparations (Swanson et al. 2004). The authors reported that the efficacy of Concerta was inferior to that of Metadate $\mathrm{CD}^{\circledR}$ (with $30 \%$ IR MPH) in the early hours after dosing. Admittedly, the daily dose of Metadate CD (20,40, or $60 \mathrm{mg}$ ) was slightly higher than that of Concerta $(18,36$, or $54 \mathrm{mg})$. In a secondary analysis on this study Sonuga-Barke et al. (2004) demonstrated that a nearly identical initial bolus of IR MPH results in similar symptom control. However, this study was conducted in an analog classroom setting and, to date, there is no study comparing the pharmacodynamic profiles of different MPH products throughout the day in a natural setting. Therefore, it is not known whether the different pharmacokinetic profiles of different extended-release MPH products result in different time course profiles in natural settings. Moreover, there has been no head-to-head comparison of the efficacy and safety of Medikinet retard with Concerta in children with ADHD. Therefore, we designed the comparison of the efficacy of Medikinet retard and Concerta trial as a randomized, double-blind, $3 \times 3$ crossover trial to investigate the effect of these two treatments in the everyday school setting and in the afternoon at home.

\section{Materials and Methods}

\section{Study design and interventions}

Comparison of the efficacy of Medikinet retard and Concerta was designed as a multicenter (nine sites), double-blind, randomized, controlled, $3 \times 3$ crossover clinical trial approved by local ethics committees. A crossover design has the advantage of being more powerful than a parallel group design. However, in the presence of "carry-over" effects, the conclusions drawn may be biased. In this study, "carry-over" effects are expected to be neglible because of the short half-life of MPH. Accordingly, each of the treatments was administered for 1 week, with assessment on school days and treatment switches on Saturdays. Since Medikinet retard (which contains equal portions of the IR pellets and the extended-release active substance enteric coated pellets) has two release stages, we decided to compare a dose of Medikinet retard with a higher IR component than Concerta (and an equivalent daily dose) and a dose of Medikinet with similar IR components in the morning as Concerta (but a lower daily dose) (Table 1), using capsules that appeared identical. Each patient received all three treatments in a sequence. Children and adolescents were randomly allocated to one of the six possible sequence combinations with stratification by dose group (low/high; Table 1). The stratification by dose group for each child and adolescent was based on her/his pretrial daily medication dose. The two randomization lists for each stratum were concealed for investigators, and the randomization sequence was created to ensure approximate balance between the sequence combinations (see Fig. 1).

In total, the length of the trial per patient was 3 weeks plus screening. The objective of this trial was to test three primary confirmatory hypotheses regarding the effects of the two drugs on patient behavior during the first three lessons in the morning at school (Fig. 2). First, we assessed whether Medikinet retard in higher IR component than Concerta (and an equivalent daily dose) is noninferior to Concerta (hypothesis I). Second, we assessed whether Medikinet retard higher IR component than Concerta (and an equivalent daily dose) is superior to Concerta (hypothesis IIa) and whether a dose of Medikinet retard with similar IR components in the morning as Concerta (but a lower daily dose) is noninferior to Concerta (hypothesis IIb). We expected a superiority of the effects of an equivalent daily dosage of Medikinet retard during the first three lessons in the morning compared with Concerta, as the morning bolus of MPH was higher in Medikinet retard. Moreover, we expected a noninferiority of Medikinet retard in a reduced daily dosage compared with Concerta retard, because the morning bolus of both drugs was similar under these conditions. Secondary exploratory hypotheses were about treatment effects on behavior in the second half of the school morning and in the afternoon at home. Due to the different pharmacokinetic profiles of both drugs, a superiority of Concerta in the late afternoon and evening was expected and tested exploratively.

\section{Inclusion and exclusion criteria}

Children participating in this trial were aged between 6:0 and 17:11 years and had a body weight $>20 \mathrm{~kg}$. Diagnosis of a Hyperkinetic Disorder according to ICD-10 (which corresponds to ADHD combined type according to Diagnostic and Statistical Manual of Mental Disorders, 4th edition (DSM-IV) (American Psychiatric Association 1994) was confirmed in a structured clinical

Table 1. Daily Dosages of Methylphenidate and Immediate Release Components of These Dosages for the Three Methylphenidate Preparations Investigated in the Comparision of the EfFicacy Of Medikinet ${ }^{\circledR}$ RETARD AND CONCERTA ${ }^{\circledR}$ TRIAL

\begin{tabular}{lccc}
\hline & $\begin{array}{c}\text { Medikinet retard with a higher IR component and an } \\
\text { approximately equivalent daily dose to Concerta }\end{array}$ & $\begin{array}{c}\text { Medikinet retard with similar IR components } \\
\text { and a lower daily dose than Concerta }\end{array}$ & Concerta \\
\hline Lower dose & $20 \mathrm{mg}(10 \mathrm{mg}$ IR) & $10 \mathrm{mg}(5 \mathrm{mg}$ IR) & $18 \mathrm{mg}(4 \mathrm{mg} \mathrm{IR})$ \\
High dose & $30 \mathrm{mg}(15 \mathrm{mg} \mathrm{IR})$ & $20 \mathrm{mg}(10 \mathrm{mg} \mathrm{IR})$ & $36 \mathrm{mg}(8 \mathrm{mg} \mathrm{IR})$ \\
\hline
\end{tabular}

$\mathrm{IR}=$ immediate release. 


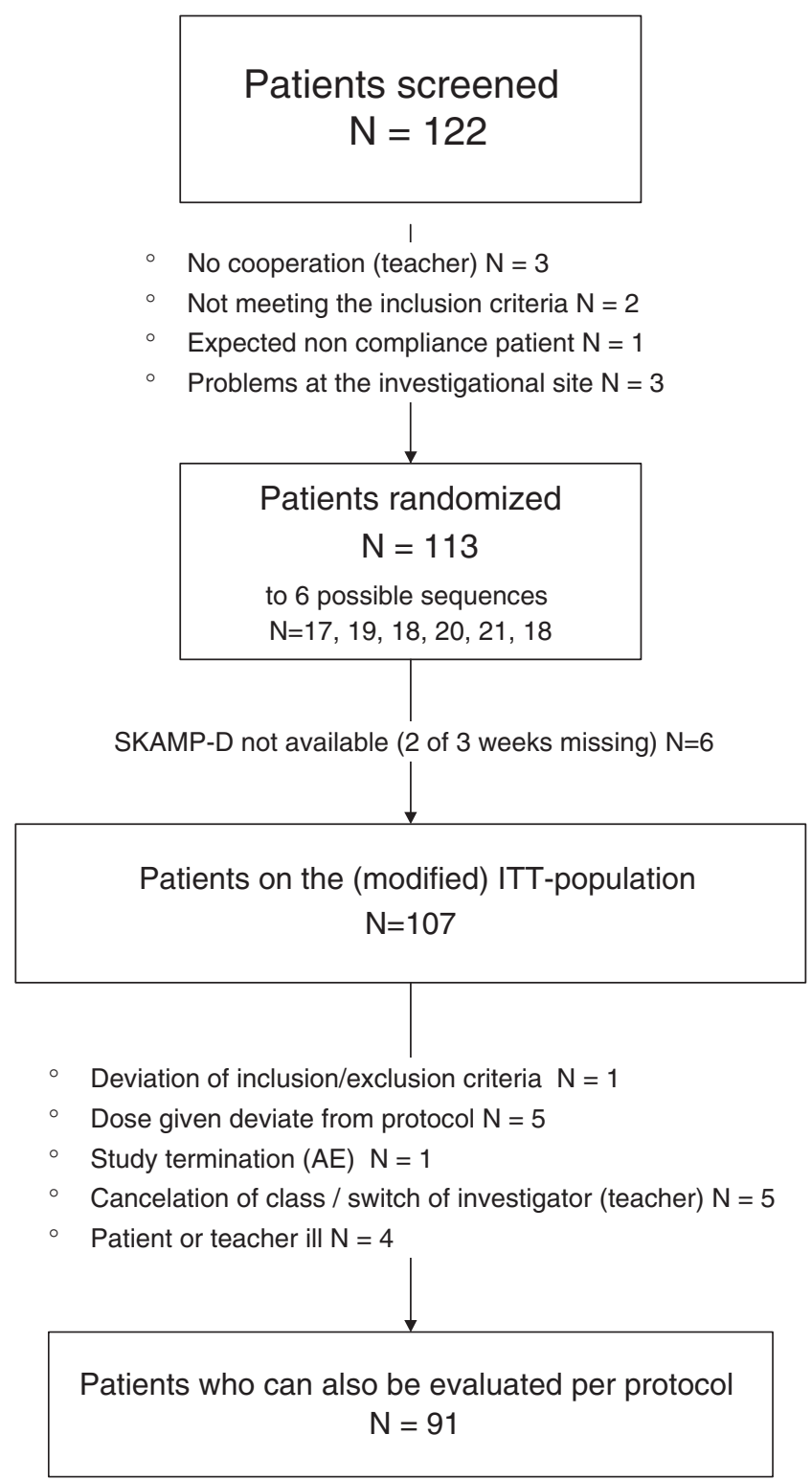

FIG. 1. Flowchart of trial participants. *One patient showed signs of clinically significant laboratory values (raised transaminases and creatine kinase values) and was, thus, excluded early from the trial by the sponsor due to safety concerns. AE, adverse events; ITT, intent-to-treat; SKAMP-D, German version of the Swanson, Kotkin, Agler, M-Flynn and Pelham scale (rated by teachers).

interview based on a German Diagnostic Checklist for ADHD (DCL-ADHD), which is a part of the German Diagnostic System for Mental Disorders in Children and Adolescents (DISYPS) (Döpfner et al. 2008b) and assesses the diagnostic criteria for ADHD according to DSM-IV and for Hyperkinetic Disorders according to ICD-10. Validity and reliability of this checklist has been established (Görtz-Dorten et al. 2008). To be included, children also had to have an intelligence quotient $\geq 80$ in a German version of the Culture Fair Intelligence tests (CFT1 or CFT20), to be attending a primary, secondary, or special school for handicapped pupils and have teacher(s) who were willing to participate and fill out rating scales. The children and adolescents were also required to respond to MPH after clinical

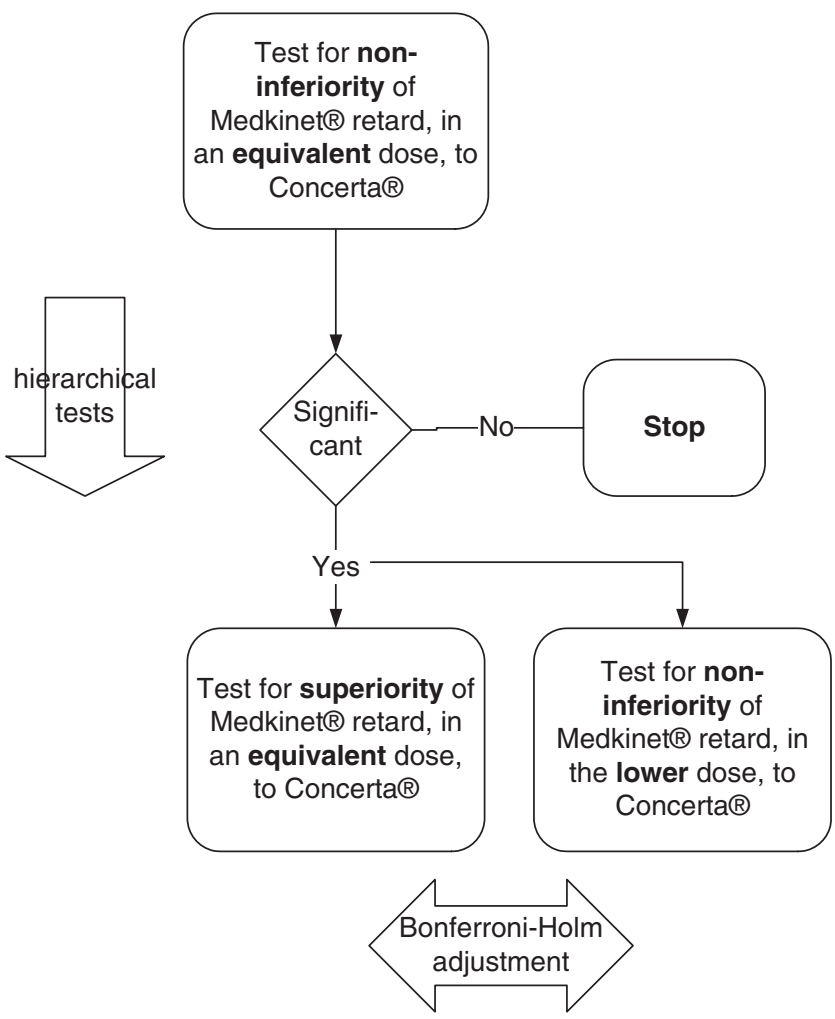

FIG. 2. Summary of confirmatory testing of hypotheses I, IIa, and IIb.

evaluation and careful titration, to take MPH at least twice-daily or a single dose of Concerta or Medikinet retard, to have received daily doses of MPH between 18 and $36 \mathrm{mg}$ before inclusion, and to have had no change in the dose of MPH in the previous month. Children and adolescents had to agree to eat breakfast everyday during the study period and had to be able to swallow the capsules. The parent/ legal guardian and patient, if applicable, gave written informed consent to participate in the study.

Children were excluded if they had a contraindication to MPH, treatment with psychostimulants other than MPH in the previous 4 weeks, or needed another ADHD treatment (e.g., behavioral therapy or immediate inpatient treatment).

For safety reasons, an electroencephalogram was performed at screening, and several laboratory tests were undertaken.

\section{Primary and secondary endpoints}

To compare Medikinet retard with Concerta in general everyday school use and in the afternoons, we assessed one primary (confirmatory) and several secondary (exploratory) outcome measures:

Swanson, Kotkin, Agler, M-Flynn, and Pelham scale-D. The primary endpoint of the confirmatory part of this trial was the total score of the German version of the Swanson, Kotkin, Agler, M-Flynn, and Pelham scale (SKAMP) (Swanson 1992; Swanson et al. 2000) (German: Fragebogen zur Verhaltensbeurteilung im Unterricht, FVU [Questionnaire for the assessment of behavior during a lesson]) (Breuer et al. 2009a) in the first 3 hours of school as assessed by the patients' teachers after each lesson. The SKAMP rating scale is a validated instrument (Wigal et al. 1998) for the assessment of attention and deportment in the classroom, which has 
been used in several trials of ADHD. The German SKAMP-D includes 10 items that describe problem behavior in the school situation. Each item is rated on a 4-point scale of impairment $(0=$ not at all; $1=$ a little; 2 = fairly severe; 3 = very severe), and a total score is obtained by dividing the sum of the item scores by the number of items answered. Teachers completed the SKAMP-D on two fixed days of the week and for a minimum of four up to a maximum of 6 hours of school each day. SKAMP-D measures were obtained at baseline (before randomization) and during weeks 1, 2, and 3. For the primary endpoint (SKAMP-D in the first 3 hours of school), a mean value was derived from the six SKAMP-D values $(2$ days $\times 3$ lesson hours). If only two SKAMP-D values or less were available, the patients' data of the primary endpoint for the respective week were set to "missing." Sensitivity analyses were performed under "worst" and "best" case scenarios in which those individuals "missing" data were replaced. In the "worst" case scenario, the individuals' worst SKAMP-D results were assigned to the Medikinet retard conditions, whereas the best results were assigned to the Concerta condition. For the "best" case scenario, this rule was inverted. As secondary endpoint, we also assessed the SKAMP-D during the later hours of school ( $4-6$ hours).

The day profile of attention-deficit/hyperactivity disorder symptoms. The day profile of ADHD symptoms (DAYAS) (Breuer et al. 2011) is a new rating scale that assesses daily profiles of ADHD and externalizing symptoms and adverse effects of ADHD medication by parent and teacher ratings. The rating scale consists of six items assessing (1) hyperactivity, (2) inattention, (3) impulsivity, (4) oppositional behavior, (5) aggressive behavior and temper tantrums, and (6) a global rating of problem behavior. Parents and teachers rate each item on a 4-point scale from $0=$ not at all to $3=$ very severe. The total score is the sum of the six item scores divided by the number of items. Parents rate the behavior of their children during four-daily periods on school days (in Germany, the children usually attend primary school in the morning only): morning, early afternoon, late afternoon, and evening. These ratings were conducted at the end of a week for the whole week and cover the typical behavior of the children at the different daily periods in that week. Additionally, potential adverse effects of ADHD medication are assessed by parents with 11 items for the whole week. The teacher form of the DAYAS comprises the same six problem items, which are assessed for the first half of the school morning and the second half of the school morning throughout the past school week. Additionally, potential adverse effects of ADHD medication are assessed by teachers with nine items for the whole week. Reliability and validity of both the parent version and the teacher version of the DAYAS have been established in several studies (Breuer et al. 2011).

FBB-ADHS. Parent and teacher ratings on a German ADHD Symptom Checklist according to ICD-10 and DSM-IV criteria (Fremdbeurteilungsbogen für Aufmerksamkeitsdefizit-/Hyperaktivitätsstörung; [Symptom checklist for attention deficit hyperactivity disorder] FBB-ADHS), which is a part of the German Diagnostic System for Mental Disorders in Children and Adolescents (DISYPS-II) (Döpfner et al. 2008b), assesses in 20 items the diagnostic criteria for ADHD according to DSM-IV and for Hyperkinetic Disorders according to ICD-10. Reliability and validity of the total scores as well as the subscale scores assessing hyperactivity-impulsivity and inattention in parent and teacher ratings have been established in several studies (Erhart et al. 2008; Döpfner et al. 2008a; Breuer et al. 2009b). The FBB-ADHS assessed ADHD symptoms in the preceding week at school (rated by teachers) and in the family (rated by parents) at the end of each week of the trial.

Both the DAYAS and the FBB-ADHS ratings from teachers and parents were obtained at the end of the week (Thursdays or Fridays) to provide a comprehensive assessment of the week and to avoid the possible impact of "carry-over" treatment effects. In addition, the teachers, the parents, and the investigators rated each week globally using the Clinical Global Impressions scale (CGI) (Collegium Internationale Psychiatriae Scalarum 1996). Adverse events (AE) including vital signs (blood pressure, heart rate), tolerance, and side effects were documented on both $\mathrm{AE}$ forms and by using the DAYAS subscale on possible side effects of drugs.

\section{Statistical methods}

The primary endpoint was evaluated for the three primary confirmatory hypotheses for which the type I error was globally controlled at a 5\% level (family-wise error rate in a strong sense). First, we compared Concerta and Medikinet retard in equivalent daily doses for noninferiority of Medikinet retard (hypothesis I) using a nonparametric test $(\alpha=0.025$, one-sided) of the intra-individual difference values with stratification (Duchateau et al. 2002). As prespecified in the statistical analysis plan, we applied the stratified exact test as proposed in detail by Duchateau and Janssen (2005). Duchateau et al. (2002) describe a new method for deriving periodadjusted nonparametric confidence intervals for pairwise treatment differences to show noninferiority or superiority. These confidence intervals do not rely on the normality assumption that often cannot be checked; moreover, they are robust against outliers, as they are based on ranks. For both clinical and statistical reasons, we chose a noninferiority margin of $\Delta=0.167$ units of the SKAMP-D in the first 3 hours of school. After rejection of null hypothesis I (the noninferiority of Medikinet retard in the equivalent daily dose to Concerta with a noninferiority margin of $\Delta=0.167$ ) at $\alpha=0.025$ (onesided), we hierarchically tested hypothesis IIa (the superiority of Medikinet retard over Concerta in equivalent daily doses) and then hypothesis IIb (the noninferiority of Medikinet retard in the reduced daily dose to Concerta with a noninferiority margin of $\Delta=0.167$ ) according to Maurer et al. (1995)—starting with $\alpha=0.025$ (onesided) and then applying the Bonferroni-Holm procedure.

The confirmatory analyses of the primary endpoint were performed using the intent-to-treat (ITT) population, which consisted of all children and adolescents who had taken the investigational drug MPH at least once and for whom data were available to allow for pairwise intra-individual comparisons of the primary endpoint (data on SKAMP-D for at least 2 weeks). Additional exploratory sensitivity analyses of the primary endpoint were performed under "best" and "worst" case scenarios (see above) and for the perprotocol (PP) population. All secondary endpoints and tolerance data were descriptively evaluated according to the methodology of the primary endpoint. Where appropriate, moreover, we performed sensitivity analyses in predefined subgroups (SKAMP-D at baseline, dose group, age group, and gender).

To determine the sample sizes, we used the parametric evaluation of a two-period design though a nonparametric test was used. To detect an effect of 0.167 units of the SKAMP-D in the first 3 hours of school given a standard deviation (SD) of the difference of 0.5 and requiring $\alpha=0.025$ (two-sided; due to Bonferroni-Holm method) and $1-\beta=0.84, n=97$, children and adolescents needed to be randomized. Taking into consideration a drop-out rate of $20 \%$, 
Table 2. Baseline Characteristics of All 113 Children and Adolescents Randomized to Treatment

\begin{tabular}{lrrrrrr}
\hline Variable & $\mathrm{n}$ & Mean & SD & Median & Minimum & Maximum \\
\hline Age, years & 113 & 10.2 & 2.3 & 10.0 & 6.0 & 16.0 \\
Weight, kg & 113 & 38.4 & 12.4 & 35.0 & 20.2 & 86.2 \\
Height, cm & 113 & 145.5 & 14.2 & 143.0 & 121.0 & 183.0 \\
BMI, kg/cm & 113 & 17.7 & 3.1 & 17.1 & 12.2 & 26.9 \\
\hline
\end{tabular}

$\mathrm{BMI}=$ body mass index $\mathrm{SD}=$ standard deviation .

122 children and adolescents were screened. The increased power was chosen to address the nonparametric test situation.

\section{Results}

A total of 122 children and adolescents were screened, and 113 were randomized to treatment (see Fig. 2 for patient disposition in the study).

Of the 113 children and adolescents randomized to treatment, 69 $(61 \%)$ had a disturbance of activity and attention (F 90.0) according to ICD-10, which corresponds to ADHD combined type according to DSM-IV, and 41 (36\%) children and adolescents had an additional Oppositional Defiant Disorder or Conduct Disorder classified according to ICD-10 (for the remaining three children and adolescents, no detailed data were available). The mean duration of ADHD for the 113 children and adolescents was 4.0 years (SD 3.7), and their average intelligence quotient was 99.2 (SD 9.7) (Table 2).

At baseline, 59 children and adolescents received MPH in an IR form at least twice per day, 48 received MPH MR, and for the remaining six patients we could not determine whether they received a delayed-release form of MPH or not. Of the 113 children and adolescents randomized, 107 were included in the ITT analysis (see above for the modified definition of the ITT-population), whereas 91 patients were evaluated as the PP-population. In the tables, where the $n$-values are smaller than these populations, data were "missing" for the particular variable. Table 3 summarizes the available primary endpoint data (SKAMP-D global scores in the first 3 hours of school, rated by teachers) by MPH treatment.

The lowest median SKAMP-D global scores were observed at baseline and for the Medikinet retard group with a higher IR component than Concerta (and an equivalent daily dose). Analysis of the SKAMP-D teacher ratings during the first 3 hours of school in the crossover design revealed that Medikinet retard with a higher IR component was noninferior and was even superior to Concerta (hypothesis I and IIa; Table 4). Moreover, we also demonstrated the noninferiority of Medikinet retard with similar IR components in the morning as Concerta (but a lower daily dose) in comparison to Concerta (hypothesis IIb; Table 4) for a noninferiority margin of $\Delta=0.167$ units of SKAMP-D. The same conclusions were obtained for analyses under the "best" and "worst" case scenarios and for the PP-population.

There was no evidence for site effects of the investigational drugs (data not shown). Table 5 summarizes the SKAMP-D global scores for the other prespecified subgroups; values were lowest in the 10-12-year-old age-group and were lower in girls as compared with boys. In children with a high baseline SKAMP-D global score $(\geq 1)$, the average improvement was descriptively stronger than in children with a lower baseline value.

The SKAMP-D global scores during the later hours of the school day (hours 4-6) are given in Table 6 and are similar to those in the first 3 hours (Table 3 ). These results support the claim provided for the primary confirmatory analysis with descriptive $p$-values of $p<0.0001$ (hypothesis I), $p=0.0006$ (hypothesis IIa), and $p<0.0001$ (hypothesis IIb). Note that this secondary outcome was not calculated if values for only 1 hour of the possible 3 hours were available. As a result, data were "missing" for this secondary outcome for more than $30 \%$ of children and adolescents.

Figure 3 shows the mean SKAMP-D global scores after each hour of school lessons; they were always lowest for Medikinet retard with a higher IR component than Concerta (and an equivalent daily dose).

Figure 4 summarizes the average daily course of DAYAS teacher and parent ratings by treatment group. An exploratory analysis appropriate for the crossover design (Duchateau et al. 2002) revealed no evidence for superiority between Medikinet retard with a higher IR component than Concerta (and an equivalent daily dose) and Concerta in the ITT-population of the DAYAS teacher rating in the early morning $(p=0.085)$ or in the teacher rating of the late morning $(p=0.172)$. The same was found for the DAYAS parent ratings in the early $(p=0.409)$ or late afternoon $(p=0.311)$ or in the evening $(p=0.696)$. The DAYAS results were similar when we analyzed the PP-population (data not shown).

The FBB-ADHS total score data are summarized in Table 7. An exploratory analysis appropriate for the crossover design (Duchateau et al. 2002) revealed some evidence for differences between Concerta and Medikinet retard with a higher IR component than Concerta (and an equivalent daily dose) for the FBB-ADHS teacher ratings in both the ITT-population $(p=0.024)$ and the PP-population $(p=0.016)$. In each analysis, the period unadjusted median difference in FBB-ADHS teacher ratings was 0.05 (minimum: -1.75 ; maximum: 1.35) in favor of Medikinet retard, which cannot be directly inferred from Table 7 , as this requires the calculation of

Table 3. Swanson, Kotkin, Agler, M-Flynn, and Pelham Scale-D Global Scores During the First 3 Hours of School at Baseline and During Treatment with the Three Formulations of Methylphenidate in the Intent-To-Treat Population

\begin{tabular}{|c|c|c|c|c|}
\hline & $\mathrm{n}^{\mathrm{a}}$ & Mean & $S D$ & Median \\
\hline Baseline & 102 & 0.61 & 0.49 & 0.45 \\
\hline Medikinet retard with a higher IR component and equivalent daily dose to Concerta & 99 & 0.60 & 0.56 & 0.44 \\
\hline Medikinet retard with similar IR components and reduced daily dose & 104 & 0.67 & 0.55 & 0.59 \\
\hline Concerta & 101 & 0.76 & 0.54 & 0.72 \\
\hline Differences ${ }^{\mathrm{b}}$ Medikinet retard in equivalent daily dose-Concerta & 93 & -0.15 & 0.44 & -0.08 \\
\hline Differences ${ }^{\mathrm{b}}$ Medikinet retard in reduced daily dose-Concerta & 98 & -0.07 & 0.42 & -0.03 \\
\hline
\end{tabular}

${ }^{a} n$ is the number of ADHD children and adolescents with complete data for the relevant variable.

b Individual difference ignoring period effects.

$\mathrm{ADHD}=$ attention-deficit/hyperactivity disorder; SKAMP-D=German version of the Swanson, Kotkin, Agler, M-Flynn, and Pelham scale. 
Table 4. Crossover Analysis of the Swanson, Kotkin, Agler, M-Flynn, and Pelham Scale-D Global Scores (Mean Value from Hours 1 to 3 of School) Comparing the Three Methylphenidate Preparations; $\mathrm{N}$ is the Number of Attention-Deficit/Hyperactivity Disorder Children and Adolescents with Complete Data on the Relevant Variables

\begin{tabular}{|c|c|c|c|c|}
\hline \multirow[b]{2}{*}{ Hypothesis tested } & \multicolumn{4}{|c|}{$\begin{array}{c}\text { p-value and effect size estimators (one-sided } 97.5 \% \\
\text { confidence interval }{ }^{\mathrm{a}} \text { ) }\end{array}$} \\
\hline & $\begin{array}{l}\text { ITT-population } \\
\quad(\mathrm{n}=101)\end{array}$ & $\begin{array}{c}\text { "Best" case scenario } \\
(\mathrm{n}=107)\end{array}$ & $\begin{array}{l}\text { "Worst" case scenario } \\
(\mathrm{n}=107)\end{array}$ & $\begin{array}{l}\text { PP-population } \\
\quad(\mathrm{n}=89)\end{array}$ \\
\hline $\begin{array}{l}\text { I. Test for noninferiority }{ }^{\mathrm{b}, \mathrm{c}} \text { of Medikinet retard } \\
\text { with a higher IR component and approximately } \\
\text { equivalent daily dose versus Concerta }\end{array}$ & $\begin{array}{c}p<0.0001 \\
(-\infty,-0.217)\end{array}$ & $\begin{array}{c}p<0.0001 \\
(-\infty,-0.242)\end{array}$ & $\begin{array}{c}p<0.0001 \\
(-\infty,-0.207)\end{array}$ & $\begin{array}{c}p<0.0001 \\
(-\infty,-0.199)\end{array}$ \\
\hline $\begin{array}{l}\text { IIa. Test for superiority }{ }^{b} \text { of Medikinet retard with } \\
\text { a higher IR component and approximately } \\
\text { equivalent daily dose versus Concerta }\end{array}$ & 0.0009 & 0.0005 & 0.0036 & 0.0004 \\
\hline $\begin{array}{l}\text { IIb. Test for noninferiority }{ }^{\mathrm{b}, \mathrm{c}} \text { of Medikinet retard } \\
\text { with similar IR components and a lower daily } \\
\text { dose versus Concerta }\end{array}$ & $\begin{array}{c}p<0.0001 \\
(-\infty,+0.051)\end{array}$ & $\begin{array}{c}p<0.0001 \\
(-\infty,+0.042)\end{array}$ & $\begin{array}{c}p<0.0001 \\
(-\infty,+0.072)\end{array}$ & $\begin{array}{c}p<0.0001 \\
(-\infty,+0.038)\end{array}$ \\
\hline
\end{tabular}

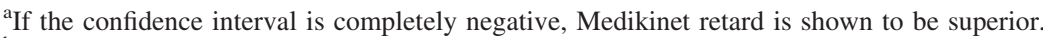

${ }^{\mathrm{b}}$ According to the method of Duchateau et al. (2002).

${ }^{\mathrm{c}} \Delta$ is the noninferiority margin, that is, Medikinet retard is allowed to be slightly worse than Concerta.

ITT $=$ intent-to-treat; $\mathrm{PP}=$ per-protocol;

individual changes. In the parental FBB-ADHS ratings, however, no such evidence for small effects was observed; for example, $p=0.308$ for the ITT-population with a period unadjusted median difference of 0.00 (minimum: -1.75 ; maximum: 1.10 ).

According to the investigators' weekly CGI ratings, 55\%, 48\%, and $56 \%$ of children and adolescents were rated as "not ill to mildly ill" for the Medikinet retard with a higher IR component than Concerta (and an equivalent daily dose), a dose of Medikinet with similar IR components in the morning as Concerta (but a lower daily dose) and Concerta groups, respectively. The corresponding values for the teacher weekly CGI ratings were $70 \%, 67 \%$, and $64 \%$, and for the parents weekly ratings were $52 \%, 41 \%$, and $53 \%$.

Overall, 199 AEs were reported; none was serious. AEs were reported for $30.6 \%, 37.7 \%$, and $35.5 \%$ of children and adolescents treated with Medikinet retard in an equivalent daily dose, Medikinet retard in reduced daily dose and Concerta, respectively. The investigator assessed $58.5 \%, 67.2 \%$, and $83.1 \%$ of the AEs as possibly, probably, or definitely having a causal relationship to the treatment.

Some of the AEs (e.g., restlessness and disturbance in attention; Table 8) may have resulted from the diminishing drug effects on ADHD. Heart rate (Table 9) was not within the reference ranges for healthy individuals, whereas the measures of blood pressure were within the reference ranges for healthy individuals.

Four children and adolescents discontinued participation in the trial because of an AE (two children and adolescents while receiving Concerta, one while on Medikinet retard in an equivalent daily dose and the other while taking Medikinet retard in a reduced daily dose). In addition to providing AE information, the investigator, parents, and teachers were asked to assess overall tolerance of the investigational drugs on a 4-point scale after every treatment stage. In about $80 \%$ of children and adolescents, tolerance was evaluated as "very good" to "good," with no evidence of differences in tolerance between the different preparations. Morover,

Table 5. Swanson, Kotkin, Agler, M-Flynn, and Pelham Scale-D Global Scores Rated by Teachers During the First 3 hours of School at Baseline and During Treatment of Children with Attention-Deficit/Hyperactivity Disorder with the Three Methylphenidate Preparations for the Prespecified Subgroups in the Intent-To-Treat Population

\begin{tabular}{|c|c|c|c|c|c|c|c|c|c|}
\hline & \multicolumn{9}{|c|}{ Subgroup } \\
\hline & \multicolumn{2}{|c|}{$\begin{array}{l}\text { Initial (baseline) } \\
\text { SKAMP-D value }\end{array}$} & \multicolumn{2}{|c|}{ Dose group } & \multicolumn{3}{|c|}{ Age, years } & \multicolumn{2}{|c|}{ Sex } \\
\hline & $\begin{array}{c}<1.0 \\
(\mathrm{n}=85)\end{array}$ & $\begin{array}{c}\geq 1.0 \\
(\mathrm{n}=20)\end{array}$ & $\begin{array}{l}\text { Lower dose } \\
\quad(\mathrm{n}=50)\end{array}$ & $\begin{array}{c}\text { High dose } \\
(\mathrm{n}=53)\end{array}$ & $\begin{array}{c}6-8 \\
(\mathrm{n}=47)\end{array}$ & $\begin{array}{c}10-12 \\
(\mathrm{n}=41)\end{array}$ & $\begin{array}{c}13-17 \\
(\mathrm{n}=17)\end{array}$ & $\begin{array}{c}\text { Male } \\
(\mathrm{n}=78)\end{array}$ & $\begin{array}{l}\text { Female } \\
(\mathrm{n}=26)\end{array}$ \\
\hline Baseline & $0.43 \pm 0.3$ & $1.35 \pm 0.4$ & $0.55 \pm 0.4$ & $0.67 \pm 0.6$ & $0.64 \pm 0.5$ & $0.54 \pm 0.5$ & $0.69 \pm 0.4$ & $0.66 \pm 0.5$ & $0.44 \pm 0.4$ \\
\hline $\begin{array}{l}\text { Medikinet retard with a } \\
\text { higher IR component } \\
\text { and equivalent daily dose }\end{array}$ & $0.46 \pm 0.4$ & $1.16 \pm 0.6$ & $0.54 \pm 0.5$ & $0.66 \pm 0.6$ & $0.61 \pm 0.6$ & $0.58 \pm 0.6$ & $0.65 \pm 0.4$ & $0.65 \pm 0.6$ & $0.46 \pm 0.6$ \\
\hline $\begin{array}{l}\text { Medikinet retard with } \\
\text { similar IR component } \\
\text { and reduced daily dose }\end{array}$ & $0.56 \pm 0.5$ & $1.13 \pm 0.7$ & $0.64 \pm 0.5$ & $0.70 \pm 0.6$ & $0.69 \pm 0.6$ & $0.62 \pm 0.5$ & $0.73 \pm 0.5$ & $0.73 \pm 0.6$ & $0.48 \pm 0.34$ \\
\hline Concerta & $0.66 \pm 0.4$ & $1.26 \pm 0.7$ & $0.75 \pm 0.5$ & $0.79 \pm 0.6$ & $0.80 \pm 0.6$ & $0.67 \pm 0.5$ & $0.91 \pm 0.5$ & $0.81 \pm 0.6$ & $0.61 \pm 0.4$ \\
\hline
\end{tabular}

Results are presented as mean $\pm \mathrm{SD} ; n$ is the number of children and adolescents with ADHD with complete data on the relevant variables. 
Table 6. Swanson, Kotkin, Agler, M-Flynn, and Pelham Scale-D Global Scores Rated by Teachers During Hours 4-6 of School at Baseline and During Treatment of Children with Attention-Deficit/Hyperactivity Disorder with the Three Methylphenidate Preparations in the Intent-To-Treat-Population

\begin{tabular}{|c|c|c|c|c|}
\hline & $\mathrm{n}^{\mathrm{a}}$ & Mean & $S D$ & Median \\
\hline Baseline & 70 & 0.71 & 0.60 & 0.60 \\
\hline Medikinet retard with a higher IR component and equivalent daily dose & 63 & 0.63 & 0.63 & 0.43 \\
\hline Medikinet retard with similar IR component and reduced daily dose & 67 & 0.71 & 0.64 & 0.50 \\
\hline Concerta & 66 & 0.80 & 0.62 & 0.66 \\
\hline Differences ${ }^{\mathrm{b}}$ Medikinet retard in equivalent daily dose-Concerta & 57 & -0.15 & 0.36 & -0.16 \\
\hline Differences ${ }^{\mathrm{b}}$ Medikinet retard in reduced daily dose-Concerta & 57 & -0.08 & 0.47 & -0.13 \\
\hline
\end{tabular}

${ }^{\text {a }} n$ is the number of children and adolescents with ADHD with complete data on the relevant variable.

${ }^{\mathrm{b}}$ Individual difference ignoring period effects.

there was no evidence of differences between the investigators', parents', and teachers' ratings (all $p$-values $>0.05$ ).

\section{Discussion}

The aim of this trial was to compare the effects of Medikinet retard with Concerta throughout the day in children and adolescents with ADHD in a natural setting. The primary outcome was ADHD symptoms in the first 3 hours of school as assessed by the patient's teachers using the classroom rating scale SKAMP-D. For the daily dose ranges investigated here, the analysis revealed that Medikinet retard with a higher IR component than Concerta and a virtually equivalent daily dose was more effective than Concerta during the first 3 hours. Moreover, a dose of Medikinet with similar IR components in the morning as Concerta but a lower daily dose was found to be noninferior to Concerta. These findings were robust in all sensitivity and exploratory subgroup analyses performed and applied similarly to all centers participating in the trial. Use of Medikinet retard in a nearly equivalent daily dosage to that of Concerta resulted in a higher initial bolus dose of IR MPH for Medikinet in the morning. Thus, noninferiority and even superiority of Medikinet retard in the first 3 hours of the morning was expected and confirmed by the results of this study. Our results in the natural setting of a regular school morning underline and confirm the findings of the COMAC study (Swanson et al. 2004), which
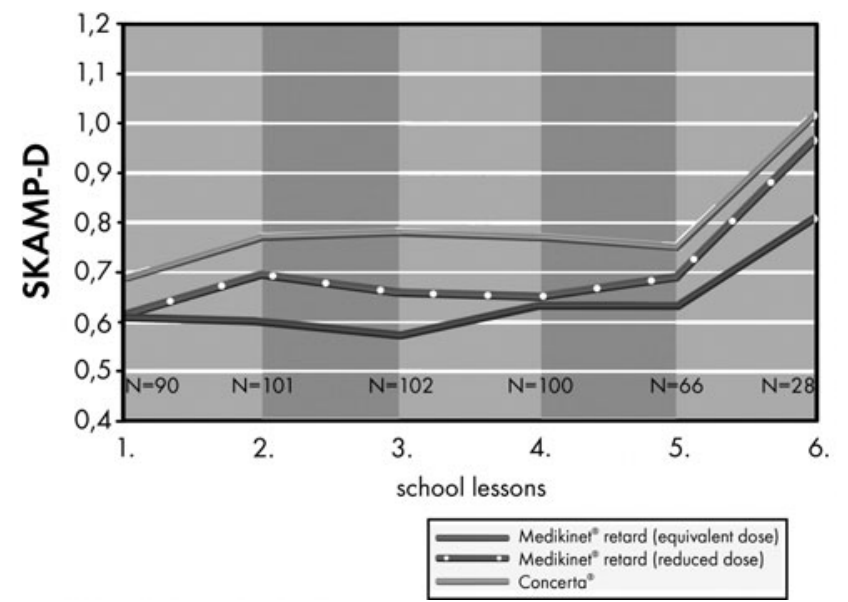

FIG. 3. SKAMP-D global score per hour of school (mean values) in children with ADHD treated with the three methylphenidate preparations. ADHD, attention-deficit/hyperactivity disorder. compared Concerta and Metadate $\mathrm{CD}$ in a laboratory classroom setting. In the COMAC study, superiority of Metadate CD with an higher initial bolus of IR MPH was found in the first 4 hours after intake using the same primary outcome measure as used in our trial. Moreover, our confirmatory finding that lower daily dosages of Medikinet retard were not inferior to higher dosages of Concerta underlines the post hoc comparisons of the COMAC study (SonugaBarke et al. 2004), which demonstrated that a nearly identical initial bolus of IR MPH results in similar symptom control. Our results are also in accordance with the findings of a smaller analog classroom study conducted by Lopez et al. (2003), which showed that $20 \mathrm{mg}$ Ritalin ${ }^{\circledR}$ LA (with 50\% IR MPH) yielded statistically greater changes than either Concerta 18 or $36 \mathrm{mg}$ on the SKAMP ratings.

In addition, the global score of the SKAMP-D assessed later in the school day (hours 4-6) indicated a similar pattern to that observed for the first 3 hours. Again, we observed evidence for the superiority of Medikinet retard with a higher IR component than Concerta and an equivalent daily dose and a noninferiority of Medikinet retard with similar IR components in the morning as Concerta but a lower daily dose regarding symptom control during the second half of the school morning. Moreover, the DAYAS

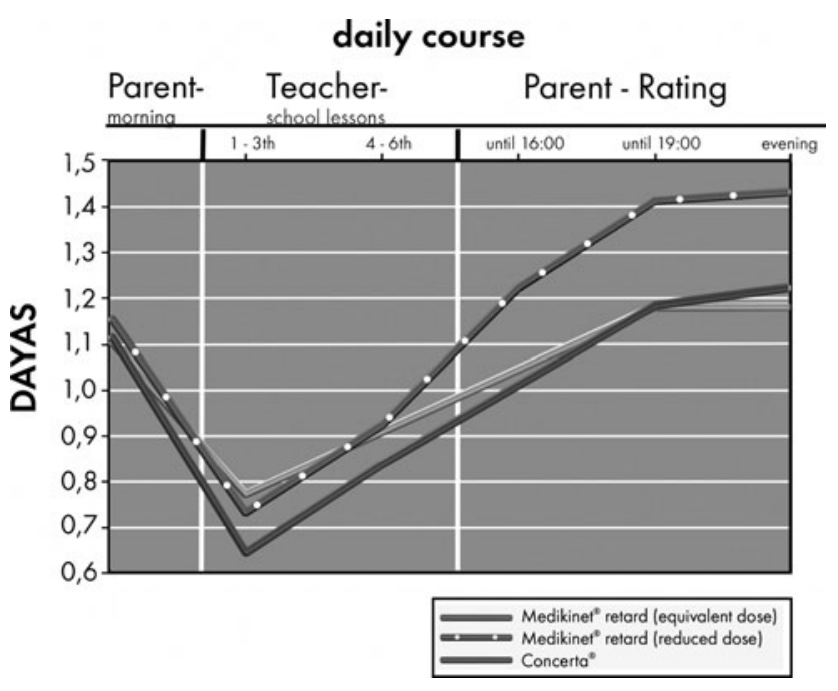

FIG. 4. DAYAS daily course (mean values) in children with ADHD treated with the three methylphenidate preparations. DAYAS, day profile of ADHD symptoms (rated by parents and teachers). 
Table 7. FBB-ADHS Global Scores Rated by Teachers and Parents at Baseline and During Treatment of Children with Attention-Deficit/Hyperactivity Disorder with the Three Methylphenidate Preparations IN THE INTENT-To-Treat-Population

\begin{tabular}{|c|c|c|c|c|c|c|c|c|}
\hline & \multicolumn{4}{|c|}{ Teachers } & \multicolumn{4}{|c|}{ Parents } \\
\hline & $\mathrm{n}^{\mathrm{a}}$ & Mean & $S D$ & Median & $\mathrm{n}^{\mathrm{a}}$ & Mean & $S D$ & Median \\
\hline Baseline & 103 & 0.76 & 0.57 & 0.60 & 105 & 1.30 & 0.60 & 1.30 \\
\hline Medikinet retard with a higher IR component and equivalent daily dose & 99 & 0.72 & 0.64 & 0.55 & 103 & 1.01 & 0.61 & 0.95 \\
\hline Medikinet retard with similar IR component and reduced daily dose & 100 & 0.73 & 0.60 & 0.55 & 104 & 1.12 & 0.70 & 0.95 \\
\hline Concerta & 98 & 0.75 & 0.59 & 0.55 & 101 & 0.98 & 0.62 & 0.90 \\
\hline Differences ${ }^{\mathrm{b}}$ Medikinet retard in equivalent daily dose-Concerta & 92 & -0.06 & 0.47 & -0.06 & 98 & 0.02 & 0.60 & 0.00 \\
\hline Differences ${ }^{\mathrm{b}}$ Medikinet retard in reduced daily dose-Concerta & 93 & -0.02 & 0.44 & 0.00 & 98 & 0.10 & 0.59 & 0.08 \\
\hline
\end{tabular}

${ }^{\mathrm{a}} n$ is the number of children and adolescents with ADHD with complete data on the relevant variables.

${ }^{\mathrm{b}}$ Individual difference ignoring period effects.

ratings of the teachers during school time and those of the parents during the afternoon and evening reveal no evidence for superiority of equivalent daily dosages of Medikinet retard and Concerta, indicating a likely similar symptom control throughout the day, whereas the efficacy of the reduced daily dosage of Medikinet retard was descriptively lower in the late afternoon and evening.

Given the differences in the release mechanisms of both products, the absence of evidence for superiority of the equivalent dosage of Concerta over Medikinet retard in the late afternoon and evening is somewhat surprising. However, in their analog classroom study (COMACS), Swanson et al. (2004) found better or similar effects of Metadate compared to with Concerta during the first 7.5 hours after intake. Concerta was superior to Metadate only at the last assessment 12 hours after intake. This result in an analog classroom was not supported in the natural setting of our study. However, in a further analysis of the COMACS trial, Sonuga-Barke et al. (2009) also report no evidence for differences between the effects of the two formulations on parent ratings of ADHD symptoms at home. This result is in line with our findings.

Unlike the SKAMP-D results, those for DAYAS gave no evidence for differences in symptom control during school time between the treatments, although the same teachers performed both ratings and there was a Spearman correlation coefficient of $r=0.7$ between ratings. It is possible that the SKAMP-D, which has also been used in international studies (e.g., Pelham et al. 2001), may be more sensitive for typical symptoms of ADHD. Moreover, the SKAMP-D might represent the individual hour of school more directly, whereas the weekly DAYAS ratings may be more likely subject to recall bias of the teacher.

From all the safety evaluations during the trial, we conclude that no symptom or event occurred that has not already been previously described in children and adolescents. There is no other specific risk to be acknowledged regarding vital signs, laboratory tests, and AEs. However, it has to be stressed that our trial included MPH responders only. Thus, the data from this trial are in accordance with those of earlier trials regarding the safety of MPH in children and adolescents.

\section{Conclusion}

From this study, it can be concluded that Medikinet retard with a higher IR component than Concerta and an equivalent daily dose was more effective than Concerta and that a dose of Medikinet with similar IR components in the morning as Concerta but a lower daily dose was found to be noninferior to Concerta in reducing ADHD symptoms during the first 3 hours in the natural setting of a regular school morning. These results can be extended to the next 3 hours of the school. Moreover, ratings of the parents during the afternoon

Table 8. Adverse Events at Baseline and During Treatment with the Three Methylphenidate Preparations in the Safety Analysis Population

\begin{tabular}{|c|c|c|c|c|}
\hline & Baseline & $\begin{array}{l}\text { Medikinet retard in } \\
\text { equivalent daily dose }\end{array}$ & $\begin{array}{l}\text { Medikinet retard in } \\
\text { reduced daily dose }\end{array}$ & Concerta \\
\hline Number of children and adolescents treated & 113 & 108 & 106 & 110 \\
\hline Number of adverse events & 32 & 41 & 61 & 65 \\
\hline Number of children and adolescents with adverse events & 24 & 33 & 40 & 39 \\
\hline \multicolumn{5}{|l|}{ Most common adverse events $(n \geq 5)$} \\
\hline Headache & 3 & 8 & 3 & 10 \\
\hline Gastrointestinal pain & 3 & 6 & 7 & 5 \\
\hline Decreased appetite & 4 & 3 & 3 & 7 \\
\hline Initial insomnia & 1 & 3 & 3 & 6 \\
\hline Aggression & 1 & 2 & 3 & 5 \\
\hline Fatigue & 2 & 0 & 4 & 3 \\
\hline Disturbance in attention & 0 & 0 & 3 & 5 \\
\hline Restlessness & 0 & 1 & 5 & 2 \\
\hline Anorexia & 3 & 0 & 0 & 4 \\
\hline Nasopharyngitis & 1 & 2 & 2 & 1 \\
\hline Weight loss & 0 & 0 & 3 & 2 \\
\hline
\end{tabular}


Table 9. Blood Pressure and Heart Rate at Baseline and During Treatment with the Three Methylphenidate Preparations in the Safety Analysis Population

\begin{tabular}{lccc}
\hline Treatment & $\begin{array}{c}\text { Frequency of cases with } \\
\text { systolic bp }>130 \mathrm{mmHg}\end{array}$ & $\begin{array}{c}\text { Frequency of cases with } \\
\text { diastolic bp }>85 \text { mmHg }\end{array}$ & $\begin{array}{c}\text { Frequency of cases with } \\
\text { heart rate }>95 \text { beats/minute }\end{array}$ \\
\hline Baseline & 0 & 3 & 3 \\
Medikinet retard in equivalent daily dose & 0 & 2 & 7 \\
Medikinet retard in reduced daily dose & 2 & 3 & 12 \\
Concerta & 1 & 3 & 10 \\
\hline
\end{tabular}

and evening indicate a likely similar symptom control throughout the day by equivalent daily dosages of Medikinet retard and Concerta. The expected superiority of Concerta in the late afternoon could not be found.

\section{Clinical Significance}

In summary, based on the efficacy and safety data from this trial, it can be claimed that Medikinet retard with a higher IR component than Concerta and an equivalent daily dose is superior to Concerta in the morning and that children and adolescents may also be treated with a lower daily dose of Medikinet retard without resulting in a clinically relevant worse effect during school time as assessed by SKAMP-D. In addition, exploratory analyses gave no evidence for superiority of equivalent daily dosages of Medikinet retard over Concerta for the DAYAS ratings of the teachers during school time and those of parents during the afternoon and evening.

\section{Disclosures}

Manfred Döpfner is currently a consultant for Medice, Shire Pharmaceutical, and Eli Lilly; has in the last 3 years received grant funding from UCB-Pharma, Medice, and Eli Lilly; has recently served on the advisory boards of Medice, Shire Pharmaceutical, and Eli Lilly; and has spoken at events sponsored by Medice, Shire Pharmaceutical, Eli Lilly, and Janssen Cilag. Claudia Ose is CEO at Center for Clinical Studies at University Hospital Essen Germany. R. Fischer is a full-time employee of Medice. R. Ammer is Chief Executive Officer of Medice. André Scherag currently serves as trial statistician on a data safety and monitoring board for Boehringer Ingelheim KG.

\section{Acknowledgments}

The authors would like to thank K-U Oehler, M. Günther, J. Gromball, B. Herpertz-Dahlmann, M. Boyraz, A. Warnke, G. Lehmkuhl, U. Schulze, J. Sinzig, and U. Behre and all the coworkers of the project, the parents, the teachers, and the children who participated in the trial.

\section{References}

American Psychiatric Association. Diagnostic and Statistical Manual of Mental Disorders, 4th ed. (DSM-IV). Washington, DC: American Psychiatric Association; 1994.

Banaschewski T, Coghill D, Santosh P, Zuddas A, Asherson P, Buitelaar J, Danckaerts M, Döpfner M, Faraone SV, Rothenberger A, Sergeant J, Steinhausen HC, Sonuga-Barke EJ, Taylor E: Longacting medications for the hyperkinetic disorders: A systematic review and European treatment guideline. Eur Child Adolesc Psychiatry 15:476-495, 2006.
Barkley RA: Attention-deficit hyperactivity disorder. Sci Am 279: 66-71, 1998.

Breuer D, Goertz-Dorten A, Rothenberger A, Döpfner M: Assessment of daily profiles of ADHD symptoms, ODD symptoms and adverse effects of ADHD medication by parent and teacher ratings. Eur Child Adolesc Psychiatry, 2011, in press.

Breuer D, Rettig K, Döpfner M: Die Erfassung von Aufmerksamkeitsund Verhaltensproblemen im Unterricht mit dem Fragebogen zur Verhaltensbeurteilung im Unterricht (FVU). [The assessment of attention and behavior problems in the classroom with the SKAMP] Diagnostica 55:11-19, 2009a.

Breuer D, Wolff Metternich T, Döpfner M: Die Erfassung von Merkmalen von Aufmerksamkeitsdefizit/Hyperaktivitätsstörungen (ADHS) anhand von Lehrerurteilen. Zur Validität und Reliabilität des FBB-HKS. [Assessment of ADHD symptoms using teacher ratings: Reliability and validity of the SCL-ADHD] Z Kinder Jugendpsychiatr Psychother 37:431-440, 2009b.

Collegium Internationale Psychiatriae Scalarum (ed): Internationale Skalen für Psychiatrie.[International Scales for Psychiatry]. Göttingen, Germany: Beltz Test; 1996.

Döpfner M, Banaschewski T, Schmidt J, Uebel H, Schmeck K, Gerber WD, Günter M, Knölker U, Gehrke M, Häßler F, Möhler E, Brünger M, Ose C, Fischer R, Poustka F, Lehmkuhl G, Rothenberger A: Lang-wirksames Methylphenidat bei Kindern mit Aufmerksamkeitsdefizit-Hyperaktivitätsstörungen. Eine multizentrische Studie.[Long-acting methylphenidate in children with ADHD-a multicenter study]. Nervenheilkunde22:85-92, 2003.

Döpfner M, Breuer D, Wille N, Erhart M, Ravens-Sieberer U, the Bella Study Group: How often do children meet ICD-10/DSM-IV criteria of attention-deficit/hyperactivity disorder and hyperkinetic disorder? Parent-based prevalence rates in a national sampleresults of the BELLA study. Eur Child Adolesc Psychiatry 17(Suppl 1):59-70, 2008a.

Döpfner M, Gerber WD, Banaschewski T, Breuer D, Freisleder FJ, Gerber-Von Müller G, Günter M, Hässler F, Ose C, Rothenberger A, Schmeck K, Sinzig J, Stadler C, Uebel H, Lehmkuhl G: Comparative efficacy of once-a-day extended-release methylphenidate, two-times-daily immediate-release methylphenidate, and placebo in a laboratory school setting. Eur Child Adolesc Psychiatry 13(Suppl 1):93-101, 2004.

Döpfner M, Görtz-Dorten A, Lehmkuhl G: Diagnostik-System für psychische Störungen nach ICD-10 und DSM-IV für Kinder- und Jugendliche (DISYPS-II). [Diagnostic system for mental disorders in children and adolescents according to ICD-10 and DSM-IV (DIYSPS-II)]. Bern, Switzerland: Huber; 2008b.

Duchateau L, Janssen P: Pairwise non-parametric non-inferiority tests in $3 \times 3$ cross-over trials: Should we adjust for period? Stat Med 24:1525-1536, 2005.

Duchateau L, Janssen P, Straetemans R, Otoul C: Adjusting pairwise nonparametric equivalence hypothesis tests and confidence intervals for period effects in $3 \times 3$ crossover trials. J Biopharm Stat 12:149-160, 2002. 
Erhart M, Döpfner M, Ravens-Sieberer U, the Bella Study Group: Psychometric properties of two ADHD questionnaires: Comparing the Conners' scale and the FBB-HKS in the general population of German children and adolescents-results of the BELLA study. Eur Child Adolesc Psychiatry 17(Suppl 1):106-115, 2008.

Faraone SV, Biederman J, Spencer TJ, Aleardi M: Comparing the efficacy of medications for ADHD using meta-analysis. MedGenMed 8:4, 2006.

Faraone SV, Buitelaar J: Comparing the efficacy of stimulants for ADHD in children and adolescents using meta-analysis. Eur Child Adolesc Psychiatry 19:353-364, 2010.

Faraone SV, Sergeant J, Gillberg C, Biederman J: The worldwide prevalence of ADHD: Is it an American condition? World Psychiatry $2: 104-113,2003$.

Findling RL, Bukstein OG, Melmed RD, Lopez FA, Sallee FR, Arnold LE, Pratt RD: A randomized, double-blind, placebocontrolled, parallel-group study of methylphenidate transdermal system in pediatric patients with attention-deficit/hyperactivity disorder. J Clin Psychiatry 69:149-159, 2008.

Görtz-Dorten A, Döpfner M: Diagnose-Checklisten aus dem Diagnostik-System für Psychische Störungen im Kindes- und Jugendalter (DISYPS-II)-Gütekriterien und klinische Anwendung. [Diagnostic checklists from the diagnostic system for mental disorders in children and adolescents (DIYSPS-II]-psychometric criteria and clinical application]. Klinische Diagnostik und Evaluation 1:378-394, 2008.

Greenhill LL, Pliszka S, Dulcan MK, Bernet W, Arnold V, Beitchman J, Benson RS, Bukstein O, Kinlan J, Mcclellan J, Rue D, Shaw JA, Stock S, Kroeger K: Summary of the practice parameter for the use of stimulant medications in the treatment of children, adolescents, and adults. J Am Acad Child Adolesc Psychiatry 40:1352-1355, 2001.

Lopez F, Silva R, Pestreich L, Muniz R: Comparative efficacy of two once daily methylphenidate formulations (Ritalin LA and Concerta) and placebo in children with attention deficit hyperactivity disorder across the school day. Paediatr Drugs 5:545-555, 2003.

Maurer W, Hothorn L, Lehmacher W: Multiple comparisons in drug clinical trials and preclinical assays: A priori ordered hypothesis. In: Biometrie in der chemisch-pharmazeutischen Industrie [Biometrics in the Chemical-Pharmaceutical Industry]. Edited by Vollmar J. Stuttgart: Fischer; 1995; pp. 3-21.

Pelham WE, Gnagy EM, Burrows-Maclean L, Williams A, Fabiano GA, Morrisey SM, Chronis AM, Forehand GL, Nguyen CA, Hoffman MT, Lock TM, Fielbelkom K, Coles EK, Panahon CJ, Steiner RL, Meichenbaum DL, Onyango AN, Morse GD: Once-aday Concerta methylphenidate versus three-times-daily methylphenidate in laboratory and natural settings. Pediatrics 107:e105, 2001.

Rösler M, Fischer R, Ammer R, Ose C, Retz W: A randomised, placebo-controlled, 24-week, study of low-dose extended-release methylphenidate in adults with attention-deficit/hyperactivity disorder. Eur Arch Psychiatry Clin Neurosci 259:120-129, 2009.
Sonuga-Barke EJS, Coghill D, DeBacker M, Swanson J: Measuring methylphenidate response in attention-deficit/hyperactvity disorder: How are laboratory classroom-based measures related to parent ratings? J Child Adolesc Psychopharm 19:891-698, 2009.

Sonuga-Barke EJS, Swanson JM, Coghill D, DeCory HH, Hatch SJ: Efficacy of two once-daily methylphenidate formulations compared across dose levels at different times of the day: Preliminary indications from a secondary analysis of the COMACS study data. BMC Psychiatry 4:28, 2004.

Stein MA, Sarampote CS, Waldman ID, Robb AS, Conlon C, Pearl PL, Black DO, Seymour KE, Newcorn JH: A dose-response study of OROS methylphenidate in children with attention-deficit/ hyperactivity disorder. Pediatrics 112:e404, 2003.

Swanson JM: School Based Assessment and Interventions for ADD Students. Irvine, CA: K.C. Publishing; 1992.

Swanson JM, Agler D, Fineberg E, Wigal S, Flynn D, Fineberg K, Quintana Y, Talebi H: University of California, Irvine, Laboratory School Protocol for pharmacokinetic and pharmacodynamic studies. In: Ritalin: Theory and Practice. 2nd ed. Edited by L.L. Greenhill, B.B. Osman. New York: Mary Ann Liebert, Inc.; 2000, pp. 405-430. Swanson JM, Wigal SB, Wigal T, Sonuga-Barke E, Greenhill LL, Biederman J, Kollins S, Nguyen AS, DeCory HH, Hirshe Dirksen SJ, Hatch SJ; COMACS Study Group: A comparison of once-daily extended-release methylphenidate formulations in children with attention-deficit/hyperactivity disorder in the laboratory school (the Comacs Study). Pediatrics 113(3Pt1):e206-e216, 2004.

Wigal SB, Gupta S, Guinta D, Swanson JM: Reliability and validity of the SKAMP rating scale in a laboratory school setting. Psychopharmacological Bull 34:47-53, 1998.

Wilens T, Mcburnett K, Bukstein O, McGough J, Greenhill L, Lerner M, Stein MA, Conners CK, Duby J, Newcorn J, Bailey CE, Kratochvil CJ, Coury D, Casat C, Denisco MJ, Halstead P, Bloom L, Zimmerman BA, Gu J, Cooper K, Lynch JM: Multisite, controlled trial of OROS methylphenidate in the treatment of adolescents with attention-deficit/hyperactivity disorder. Arch Pediatr Adolesc Med 160:82-90, 2006.

Wolraich M, Greenhill LL, Pelham W, Swanson J, Wilens T, Palumbo D, Atkins M, McBurnett K, Bukstein O, August G: Randomized, controlled trial of OROS methylphenidate once a day in children with attention-deficit/hyperactivity disorder. Pediatrics 108:883892, 2001.

Address correspondence to: Manfred Döpfner, Ph.D.

Department of Child and Adolescent Psychiatry and Psychotherapy University of Cologne Robert Koch St. 10 D-50931 Cologne Germany

E-mail: manfred.doepfner@t-online.de 